\title{
Study of the Inferior Alveolar Canal and Mental Foramen on Digital Panoramic Images
}

\author{
Carlos M Pria, Farah Masood, Joy M Beckerley, Robert E Carson
}

\section{ABSTRACT}

Objectives: To study the radiographic location of the mental foramen and appearance of the inferior alveolar canal and the relationship between image gray values and the clarity of inferior alveolar canal on the digital panoramic images and to evaluate if the histogram equalization of the digital image would improve the visualization of the inferior alveolar canal outline on the digital panoramic images in the mandible.

Methods: Five hundred digital panoramic images were evaluated by two examiners using a specific inclusion criteria. Only the right side of the mandible was studied. Chi-square analyses were used for comparisons of distributions. Mean and median pixel values were analyzed separately with a one-way analysis of variance. Also, percentages were calculated to report the usefulness of the histogram equalization for visualization of canal.

Results: Results show variation in location of mental foramen. Most frequent location of the mental foramen was reported as first and second premolar region. Chi-square analysis showed that the frequency of occurrence of the mental foramen was equally probable for any of the three locations. The study did not find significant usefulness of the gray values obtained from the histogram equalization in predicting the clarity of inferior alveolar canal outlines.

Clinical significance: Knowing the normal relationship and the anatomical variation of the maxillofacial structures for each patient is important for surgical implant treatment planning to avoid future complications. It is also important to be familiar with the advantages and limitations of diagnostic aids available before making treatment planning decisions based on such findings.

Keywords: Digital imaging, Panoramic, Inferior alveolar canal, Mental foramen.

How to cite this article: Pria CM, Masood F, Beckerley JM, Carson RE. Study of the Inferior Alveolar Canal and Mental Foramen on Digital Panoramic Images. J Contemp Dent Pract 2011;12(4):265-271.

Source of support: Nil

Conflict of interest: None declared

\section{INTRODUCTION}

The inferior alveolar canal (IAC) or the mandibular canal is an important osseous structure located in the mandible that permits the passage of the neurovascular bundle including the inferior alveolar nerve (IAN) and the mandibular division of the trigeminal nerve (V3). The IAN is vital for normal function of the sensory system in the lower third of the face. The IAC begins at the mandibular foramen, in the middle third of the ascending ramus and ends in the mental foramen usually below the apex of the second premolar or between the first and second premolars. ${ }^{1}$ As the IAN proceeds anteriorly in the mandibular canal, it traverses the mandible from the lingual to the buccal side. The path of the IAC is divided into four sectors: Ramus, angle, body and the mental sector. ${ }^{2}$

The terminal branches of the IAN exit the mandible through the mental foramen. Most often, the mental foramen is found at a more coronal position on the body of the mandible than the IAC. ${ }^{3}$ It opens onto the mandibular surface in an oblique direction and may have an anterior loop or may form a curve medially. ${ }^{4}$ Normally, three nerve branches emerging from the mental foramen innervate the skin of the mental area, the skin of the lower lip, mucous membranes and the gingiva as far posteriorly as the second premolar. $^{5}$

Radiographic images are considered a vital diagnostic aid during evaluation and treatment planning. Radiographically, the mental foramen is presented as an oval radiolucency in the premolar region; however, accurate localization of the mental foramen and the IAC on the radiographic images is not always possible. ${ }^{7,12}$

The location of the mental foramen has been studied clinically and radiographically and anatomical variations have been reported. In the horizontal plane, it is usually 
located by the apex of the second mandibular premolar or between the apices of the premolars. ${ }^{6,14,15}$ Atypically, the mental foramen can be found anteriorly close to the apex of the canine or posteriorly near the first molar $^{7}$ or along the long axis of the second premolar. ${ }^{12,13}$ In the vertical plane, the foramen can be 14 to $15 \mathrm{~mm}$ from the inferior border of the mandible. ${ }^{8}$ Another study reported the foramen was $12 \mathrm{~mm}$ from the most apical portion of the lower cortex of the mandible. ${ }^{9}$ Therefore, it can be concluded that the location of the mental foramen is not constant in the horizontal or vertical planes and its anatomy may vary.

The mental foramen can be detected on the panoramic images, but clear visibility was reported only $49 \%$ of the time. ${ }^{10,11}$ Also, on panoramic images the IAC may or may not be observed clearly. ${ }^{19,20}$ It has been reported that panoramic images do not provide reliable information about the location of the IAC. ${ }^{19}$

With the increased use of dental radiographic images ${ }^{15,16}$ and dental implants in the last 20 years, ${ }^{28}$ accurate diagnosis and treatment planning for the surgical placement of dental implants has become essential in order to avoid postoperative complications as well as paresthesia or even implant failure.

The position and appearance of anatomical structures such as the IAC and mental foramen are important considerations during the surgical treatment of the mandible. ${ }^{17}$ With implant surgery, preoperative radiographic examination is considered essential in order to avoid injury to the IAN and to obtain important data regarding quantity and quality of bone at the surgical site. ${ }^{18}$ Previous research supports the premise that bone density can be linked to the pixel value of images. ${ }^{19,20}$ A literature search revealed multiple studies indicating morphology and location of the inferior alveolar canal but very limited information was found to our knowledge on usefulness of gray values and histogram equalization, for improving the detection of this canal in the mandible. Hence, the objectives of the present study were:

1. To investigate the radiographic location of the mental foramen on digital panoramic images.

2. To study the radiographic appearance of the inferior alveolar canal on digital panoramic images.

3. To study the relationship between image gray values and the clarity of the IAC on digital panoramic images in the posterior mandible.

4. To evaluate if the histogram equalization of the digital image would improve the visualization of the IAC outline on the digital panoramic images in the posterior mandible.

\section{METHODS}

A total of 500 panoramic digital images, previously made over past 3-year period (2007-2009), were included for evaluation. The study was approved by the Institutional Review Board. The images were selected from the patient pool of the Department of Oral Diagnosis and Radiology, College of Dentistry. These images were acquired earlier on patients who presented to the College of Dentistry for various treatment needs such as initial screening or oral surgery. The images were made according to the manufacturer's operating instructions using a PlanmecaProline XC digital panoramic machine (PlanmecaOy, Helsinski, Finland) at the university by trained radiology technicians or a dental student at the College of Dentistry under the supervision of a trained radiology technician. The exposure parameters varied depending on the patient's anatomical structures (tube potential: 60-76 kV, tube current: 4-12 mA).

Since the mental foramina usually have symmetrical configuration, even among different ethnicities, ${ }^{12,14,15,22-24,28}$ only the right side of the mandible was studied.

The following inclusion criteria were used:

- Adult dentate patients who were 18 years or older with permanent dentition

- Diagnostic quality images with acceptable density and contrast

- $\quad$ Presence of the canine, first premolar, second premolar and first molar in the images

- Images with minimal positioning errors and none or minimal superimposition of structures.

Exclusion criteria were:

- Patients younger than 18 years of age

- Mixed dentition and or incomplete eruption of teeth because of the possibility of obscuring the ROI such as the region of mental foramen

- Presence of radiolucent or radiopaque lesions in the body of the mandible, anywhere in the area extending from the right third molar region to the left third molar region. During the initial evaluation, approximately 34 images were excluded for the following reasons: Periapical pathology obscuring regions of interest, missing canines, premolars and molars and poor image quality.

Prior to data collection sessions, the two examiners underwent two training sessions a week apart. For the training sessions, the examiners randomly selected 50 panoramic images that were not part of the study sample and learned the use of the imaging software. The appearance of the IAC and mental foramen was also studied. Any disagreements were discussed and the examiners were calibrated.

The images were evaluated in standard viewing conditions under dimmed lighting on a 20.1-inch Dell Model: 2007 wfp LCD monitor with a contrast ratio: 800:1 and optimal preset resolution: $1680 \times 1050$ at $60 \mathrm{~Hz}$ (Dell Inc, Round Rock, TX). 
The examiners identified the location of the mental foramen on the panoramic images according to tooth location as follows:

- Canine

- Canine-First premolar (Can-1preM)

- First premolar-Second premolar (1preM-2preM)

- Second premolar-First molar (2preM-1M).

Next, the examiners identified the appearance and visibility of the IAC on all images according to the following criteria:

Type I: Visible superior and inferior borders (welldefined Fig. 1).

Type II: Visible superior and invisible inferior borders (partially defined Fig. 2).

Type III: Visible inferior and invisible superior borders (partially defined Fig. 3).

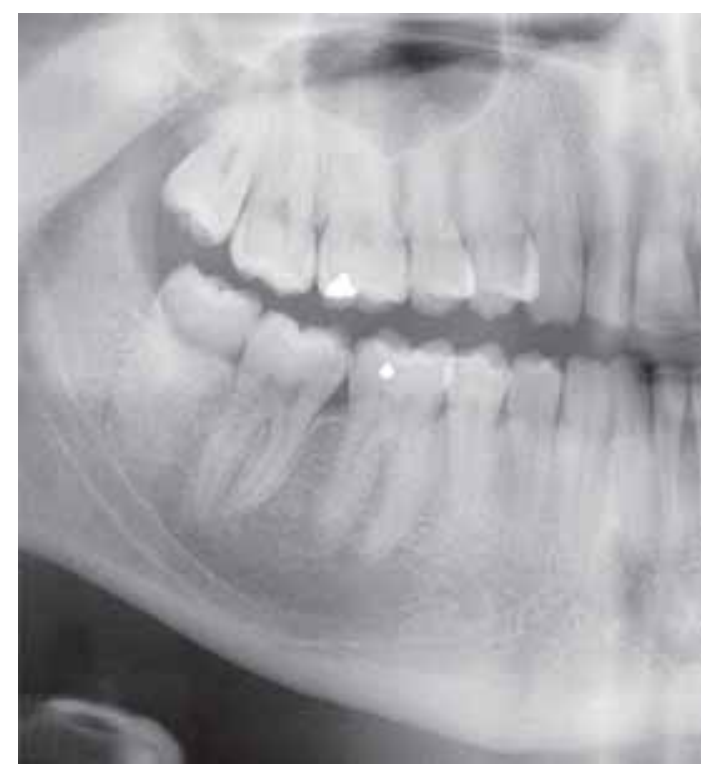

Fig. 1: Visible superior and inferior borders of IAC (well-defined)

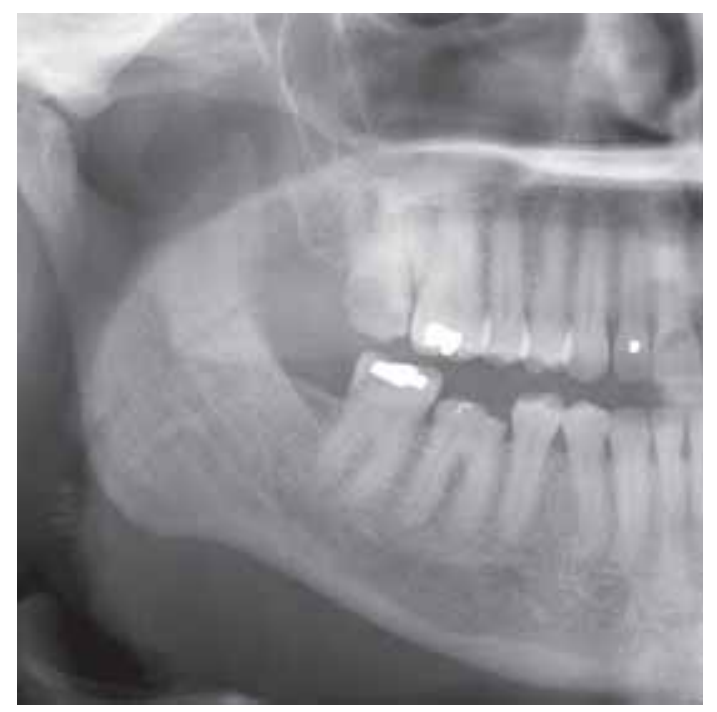

Fig. 2: Visible superior and invisible inferior borders of IAC (partially defined)
Type IV: Invisible superior and inferior borders (not defined Fig. 4).

Then, the examiners determined the gray values of the bone in the apical region of the following locations (according to tooth) using the software provided with the panoramic machine:

- Canine

- Canine-First premolar

- First premolar-Second premolar.

To record the gray values, ROI was drawn in the apical region. The size of the ROI did not exceed $5 \times 5 \mathrm{~mm}$ for each location. A minimum distance of $2 \mathrm{~mm}$ was kept between the teeth and the ROI. Teeth were not included in the ROI. Also, any suspected periapical pathology, large bone marrow spaces or anatomical features, such as

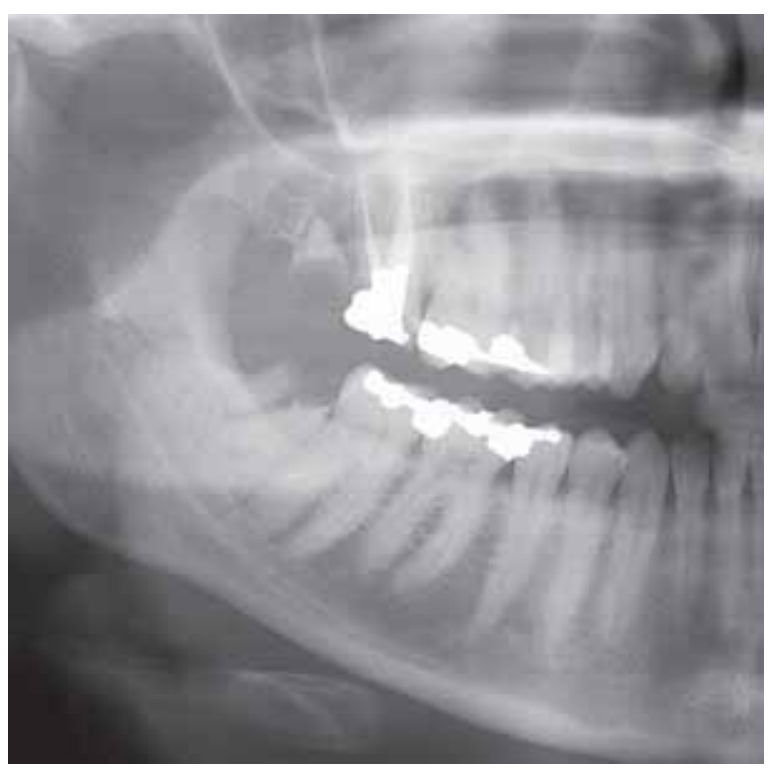

Fig. 3: Visible inferior and invisible superior borders of IAC (partially defined)

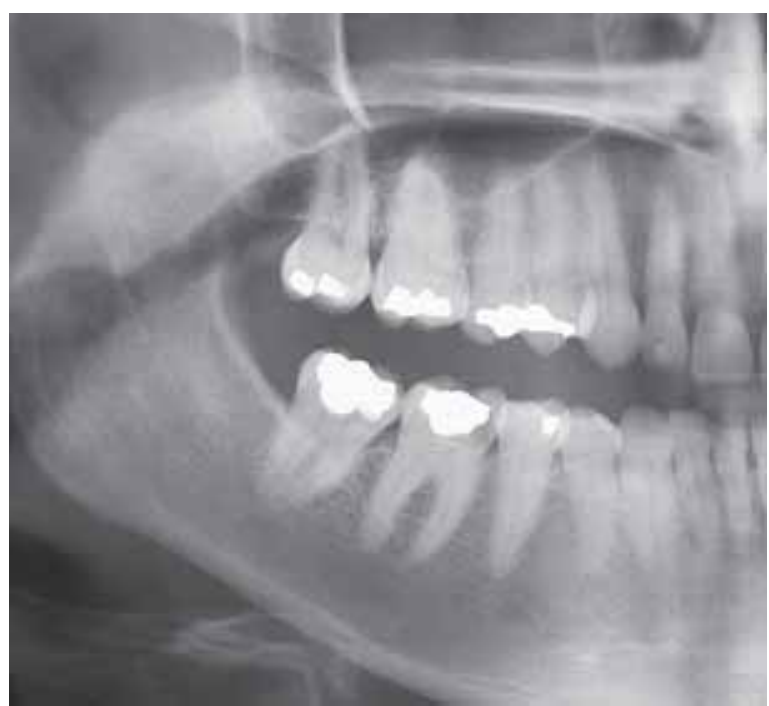

Fig. 4: Invisible superior and inferior borders of IAC (not defined) 
the mental foramen or the IAC, were not included in the ROI.

At last, one of the examiners performed the histogram equalization of right body of the mandible as ROI using the software provided with the panoramic machine.

The ROI was drawn on the digital image to include the body of the mandible from the mesial of canine to the distal of the first molar region and histogram equalization was performed. The examiner recorded whether the use of this enhancement improved the visualization and detection of the cortical outline of the IAC or not on the following scale: (1) Improved appearance of IAC, (2) not sure and (3) did not improve the appearance of IAC.

\section{Statistical Analysis}

Data was subsequently put on Excel spreadsheets. Analyses of the data were performed using SAS version 9.1. Frequency data were summarized and Chi-square analyses were used for comparisons of distributions. The mean and median pixel values were analyzed separately with a oneway analysis of variance. Also, percentages were calculated to report the usefulness of the histogram equalization for visualization of canal.

\section{RESULTS}

\section{Location of Canal}

To examine the most common location of the mental foramen, the frequencies and percentages of each identified location were calculated.

The mental foramen was most frequently located in the '1preM-2preM' location (59\% ). The second most frequent location of the mental foramen was the '2preM-1M' area (36\%). The foramen was present in the 'Can-1preM' location only $5 \%$ of the time. None was identified in the canine location. The mental foramen could not be identified in 36 digital images (7\%), as the outlines were not corticated and nonidentifiable on these images.

Next, a Chi-square analysis was used to determine if there was an equal chance for the mental foramen to be identified in any of the three identified locations: Can1preM, 1preM-2preM and 2preM-1M. The Chi-square analysis showed that the frequency of occurrence of the mental foramen was equally probable for any of the three locations. To summarize, the results of Chi-square showed that the mental foramen was located in the '1preM-2preM' location significantly more often $\left[\chi^{2}(2)=206.5\right.$, $\left.\mathrm{p}<0.0001\right]$ than the 'Can-1preM' location or the '2preM-1M' location. The frequency of location of the mental foramen is shown in Table 1.
Table 1: Frequency of the location of the mental foramen

\begin{tabular}{lcc}
\hline Location & Frequency & Percent \\
\hline Not identified & 36 & 7.20 \\
Canine & 0 & 0.00 \\
Can-1preM & 23 & 4.60 \\
1-2preM & 275 & 55.00 \\
2preM-M & 166 & 33.20 \\
\hline
\end{tabular}

Note: Can-1preM: Canine and 1st premolar; 1-2preM: 1st and 2nd premolar; 2preM-M: 2nd premolar and 1st molar

\section{Type of Canal}

In $36.75 \%$ of cases, the inferior alveolar canal was classified as type I (well-defined superior and inferior cortical outline). The second most common appearance of the IAC was type III (partially defined, visible inferior and invisible superior borders) in $34.14 \%$ of the images. The IAC was not defined (type IV, invisible superior and inferior borders) in 28.92\% of the images and only one was identified as a type II (partially defined, visible superior and invisible inferior borders). The frequency of the appearance of the IAC is shown in Table 2.

\begin{tabular}{lcc}
\multicolumn{4}{c}{ Table 2: Frequency of the appearance of the IAC } \\
\hline Type & Frequency & Percent \\
\hline Type I canal defined & 183 & 36.75 \\
Type II canal partially defined (superior) & 1 & 0.20 \\
Type III canal partially defined (inferior) & 170 & 34.14 \\
Type IV not defined & 144 & 28.92 \\
\hline
\end{tabular}

\section{Gray Values and Appearance of the IAC}

ANOVA was used to analyze the differences in mean and median gray values at the three different levels of identification. Separate analyses were performed for the mean and median gray value at each of the three locations. There was no significant relationship in the mean gray values at the three sites and the appearance of the IAC (Table 3). There was no significant relationship in the median gray values at the three sites and the appearance of IAC (Table 4).

\section{Histogram Equalization Usefulness}

The findings were found as follows: Out of 500 images, the canal visualization improved on 227 cases (45\%), 189 images (37\%) did not show any improvement and on 84 (16.4\%) images the examiner was not sure.

\section{DISCUSSION}

Since, the location of the mental foramen can change during the development of the jaws, ${ }^{28}$ this study utilized panoramic images taken on patients whose growth and development was completed. Additionally, the evaluation was done only 
Study of the Inferior Alveolar Canal and Mental Foramen on Digital Panoramic Images

\begin{tabular}{lcccccc}
\hline \multicolumn{7}{c}{ Table 3: Relationship between mean gray values and appearance of the IAC } \\
\hline Site & $F$ & DF & $p$-value & Type / $^{*}$ & Type $^{*}{ }^{*}$ & Type IV* $^{*}$ \\
\hline Canine & 2.57 & 2.494 & 0.078 & $130.30(29.28)$ & $123.93(28.24)$ & $124.73(28.26)$ \\
Can-1PreM & 1.09 & 2.494 & 0.338 & $126.59(27.80)$ & $122.79(28.84)$ & $126.79(26.97)$ \\
1-2PreM & 1.25 & 2.494 & 0.287 & $105.43(31.19)$ & $102.48(30.17)$ & $107.87(29.10)$ \\
\hline
\end{tabular}

*Means and standard deviations

\begin{tabular}{lcccccc}
\multicolumn{7}{c}{ Table 4: Relationship between median gray values and appearance of the IAC } \\
\hline Site & $F$ & DF & $p$-value & Type $I^{*}$ & Type $I^{*}$ & ${\text { Type } I V^{*}}^{\text {(2) }}$ \\
\hline Canine & 2.49 & 2.494 & 0.084 & $130.23(29.38)$ & $123.95(28.39)$ & $124.72(28.36)$ \\
Can-1PreM & 1.12 & 2.494 & 0.327 & $126.52(27.97)$ & $122.64(28.98)$ & $126.71(27.09)$ \\
1-2PreM & 1.05 & 2.493 & 0.351 & $105.41(31.22)$ & $102.90(29.46)$ & $107.83(29.19)$ \\
\hline
\end{tabular}

Note: Can-1preM = Canine and 1st premolar; 1-2preM: 1st and 2nd premolar; 2preM-M: 2nd premolar and 1st molar, * Means and standard deviations

on panoramic images with canines, premolars and molars present. The mental foramen is frequently described in the literature as situated in the region of the second premolar $7,12,21,24,26$ or between the first and second premolar ${ }^{14,15,23,25}$ in the fully developed mandible.

The first objective to be addressed was to identify the location of the mental foramen. In our investigation, in a sample size of 500 panoramic images, we found the mental foramen was positioned between the long axis of the first and second premolar (59\% of the time). The results of our study were similar to the result of many other studies. ${ }^{3,14,15,23,24}$ In $33 \%$ of the cases, the mental foramen was found as far posteriorly as the apices of the first molars.

The possibility of location of the mental foramen as far posterior as the apices of the first molar (33\%) is of clinical significance as this may be interpreted incorrectly as periapical pathology on radiographic images.

The next objective addressed the identification of the IAC. Our study demonstrated that in many panoramic images, we were able to identify the outline and the location of the IAC: Type I defined 36.75\%, followed closely by a partially defined canal (type III, partially defined inferior border $34.14 \%$ ). The finding that over $60 \%$ of the inferior alveolar canals could not be identified in digital panoramic images, may suggest the use of more advanced diagnostic modalities for accurate identification of the mental foramen and the IAC (i.e. cone-beam computer tomography) before invasive surgical procedures.

The third objective of our study was to examine the differences in gray value at the previously described types/ appearances of IAC (type I, type II, type III and type IV) in three different locations. There was no significant relationship between the mean gray values at the three sites (Can, Can-1PM, 1PM-2PM) and the appearance of the IAC. The higher gray values did not indicate a more defined and detectable outline of IAC. This last finding differs with the study by Naitoh (2009), which found a relationship between the pixel value at three different locations and the depiction of the mandibular canal. However, in the Naitoh (2009) study, the pixel value was measured in cone-beam computer tomography as compared to our study in which the values were measured on the digital panoramic images. We were not able to confirm a relationship between gray values and the depiction of the IAC outline in the mandible on the 500 digital panoramic images evaluated in this study.

Our last objective was to assess the usefulness of histogram equalization. Histogram equalization maximizes the image contrast by applying a gray level transformation, which flattens the resulting histogram. The global image contrast is improved. This allows for areas of lower local contrast to gain a higher contrast. Histogram equalization accomplishes this by effectively spreading out the most frequent intensity values. ${ }^{29}$ This study found that, in some cases, the histogram equalization tool provided by the manufacturer improved the visualization of the cortical outline of the IAC but not found very relevant for everyday clinical practice. Therefore, more research is needed to evaluate the effectiveness of histogram tools for clinical applications.

\section{Possibilities for Future Studies}

Future studies may be done to include variables such as age, gender and medical history and study the relationship of these factors to the radiographic definition of the IAC. Both sides of the mandible may be studied for evaluation of the mental foramen location. Additionally, it may be helpful to include multiple examiners with different levels of expertise. Interexaminer and intraexaminer reliability may be evaluated.

\section{CONCLUSION}

We found that in majority of the cases, mental foramen was positioned between the long axis of the first and second premolar. In limited cases, this foramen may be located as 
far back as 1st molar region. So, the clinicians have to perform a thorough radiographic interpretation. Knowing the location of IAC is of utmost importance when dental implants are treatment of choice. At last, when utilizing digital image enhancement tools for diagnostic purposes, one must be trained and know the limitations of such applications. Often these tools may provide very limited additional information for clinical use.

\section{CLINICAL SIGNIFICANCE}

It is essential to acquire accurate anatomical information about the location of the associated structures prior to implant surgery. It is also important to have a good understanding of the anatomical variations in location and shape of maxillofacial structures to aid in better treatment planning and hence help improve the predictability and outcome of surgical procedures. The outline of IAC is not always corticated and thus not easily detected. Various digital image enhancement tools are available to aid in such cases. We think that the practicing dentist must have a clear understanding of such tools provided with the imaging software before utilization as improper use may lead to treatment impediments and failure. This study emphasizes the importance of recognizing and understanding the limitations of these tools. Although description of the course and location of IAC and mental foramen on panoramic images is very useful in everyday dental practice but one has to recognize the limitations of this modality. Depending on the complexity of the case and professional judgment of the dentist, use of advance imaging such as cone-beam computed tomography may be considered, if conventional techniques provide none or limited vital information. The authors think that the findings of this study will further aid clinicians in better understanding of the mandibular anatomical variations, and use of digital enhancement tools during treatment planning of the surgical procedures.

\section{REFERENCES}

1. Misch CE. Applied anatomy for dental implants. In: Misch CE (Ed). Contemporary Implant Dentistry (3rd ed). St Louis, Mo: Mosby 2005:495-500.

2. Poirot G, Delattre JF, Palot C, Flament JB. The inferior alveolar artery in its bony course. Surg Radiol Anat 1986;8:237-44.

3. Fishel D, Buchner A, Hershkowith A, Kaffe I. Roentgenologic study of the mental foramen. Oral Surg Oral Med Oral Pathol 1976;41:682-86.

4. Arzouman MJ, Otis L, Kipnis V, Levine D. Observations of the anterior loop of the inferior alveolar canal. Int J Oral Maxillofac Implants 1993;8:295-300.

5. Kieser JA, Paulin M, Law B. Intrabony course of the inferior alveolar nerve in the edentulous mandible. Clin Anat 2004; 17:107-11.
6. Phillips JL. The mental foramen: 1 . Size, orientation and positional relationship to the mandibular second premolar. J Endod 1990;21:340-42.

7. Ngeow WC, Yuzawati Y. The location of the mental foramen in a selected Malay population. J Oral Sci 2003;45:171-75.

8. Agthong S, Huanmanop T, Chentanez V. Anatomical variations of the supraorbital, infraorbital, and mental foramina related to gender and side. J Oral Maxillo fac Surg 2005;63:800-04.

9. Neiva RF, Gapski R, Wang HL. Morphometric analysis of implant-related anatomy in Caucasian skulls. J Periodontol 2004; 75:1061-67.

10. Jacobs R, Mraiwa N, Van Steenberghe D, Sanderink G, Quirynen M. Appearance of the mandibular incisive canal on panoramic radiographs. Surg Radiol Anat 2004;26:329-33.

11. Yosue T, Brooks SL. The appearance of mental foramina on panoramic radiographs. I. Evaluation of patients. Oral Surg Oral Med Oral Pathol 1989;68:360-64.

12. Al Jasser NM, Nwoku AL. Radiographic study of the mental foramen in a selected Saudi population. Dentomaxillofac Radiol 1998;27:341-43.

13. Al-Talabani N. Precise computer-based localization of the mental foramen on panoramic radiographs in a Kurdish population. Oral Radiol 2008;24:59-63.

14. Haghanifar S. Radiographic evaluation of the mental foramen in a selected Iranian population. Indian J Dent Res 2009; 20(2):150-52.

15. Gungor K. A radiographic study of location of mental foramen in a selected Turkish population on panoramic radiograph. Coll Antropol 2006;30(4):801-05.

16. Misch CE. Diagnostic imaging and techniques. In: Misch CE ed. Contemporary Implant Dentistry (3rd ed). St Louis, Mo: Mosby; 2005:44-45.

17. Bolin A. Radiographic evaluation of mandibular posterior implant sites: Correlation between panoramic and tomographic determinations. Clin Oral Implants Res 1996;7(4):354-59.

18. Dula K. The radiographic assessment of implant patients: Decision-making criteria. Int J Oral Maxillofac Implants 2001; 16(1):80-89.

19. Angelopoulos C, Thomas SL, Hechler S, Parissis N, Hlavacek M. Comparison between digital panoramic radiography and conebeam computed tomography for the identification of the mandibular canal as part of presurgical dental implant assessment. Journal of Oral and Maxillofacial Surgery 2008; 66(10):2130-35.

20. Naitoh M. Relationship between cancellous bone density and mandibular canal depiction. Implant Dent 2009;18(2):112-18.

21. Amorin M. The mental foramen position in dentate and edentulous Brazilian's mandible. Int J Morphol 2008; 26(4): 981-87.

22. Captier G, Lethuilier J, Oussaid M, Canovas F, Bonnel F. Neural symmetry and functional asymmetry of the mandible. Surg Radiol Anat 2006; 28:379-86.

23. Olasoji HO, Tahir A, Ekanem AU, Abubakar AA. Radiographic and anatomic locations of mental foramen in northern Nigerian adults. Niger Postgrad Med 2004;11:230-33.

24. GremmerTebo H. An analysis of the variations in position of the mental foramen. The Anatomical Record 1950;107(1): 61-66.

25. Moiseiwitsch J. Position of the mental foramen in a North American, white population. Oral Surg Oral Med Oral Pathol Oral Radiol Endod 1998;85(4):457-60.

26. Kim IS. Position of the mental foramen in a Korean population: A clinical and radiographic study. Implant Dent 2006;15(4):404-11. 
27. Smartt J. The pediatric mandible: I. A primer on growth and development. Plastic and Reconstructive Surgery 2005; 116(1):14e-23e.

28. Cochran DL. Implant therapy. Ann Periodontol 1996;1:707-90.

29. http://en.wikipedia.org/wiki/Histogram_equalization.

\section{ABOUT THE AUTHORS}

\section{Carlos M Pria}

Private Practice, 13262 SW 29th Terrace, Miami, Florida 33175, USA

\section{Farah Masood (Corresponding Author)}

Diplomate, American Board of Oral and Maxillofacial Radiology, Associate Professor and Director of Radiology, Department of Oral Diagnosis and Radiology, University of Oklahoma College of Dentistry, 1201 N Stonewall Avenue, Oklahoma City, OK
73117, USA, Phone: 405-271-5988, Fax: 405-271-3158, e-mail: farah-masood@ouhsc.edu

\section{Joy M Beckerley}

Diplomate, American Board of Periodontology, Clinical Associate Professor, Graduate Periodontics Program, University of Oklahoma College of Dentistry, 1201 N Stonewall Avenue, Oklahoma City, OK 73117, USA

\section{Robert E Carson}

Diplomate, American Board of Periodontology, Professor, College of Dentistry, Chair, Department of Periodontics, Chair, Division of Oral Biology, Director, Graduate Periodontics Program, University of Oklahoma College of Dentistry, 1201 N Stonewall Avenue Oklahoma City, OK 73117, USA 